Received: 16.06 .2020

Revised: 29.07.2020

Accepted: 21.08 .2020

DOI: $10.17804 / 2410-9908.2020 .4 .048-061$

\title{
APPLYING THE METHOD OF PLANE SECTIONS FOR EVALUATING THE PARAMETERS OF FLIGHT VEHICLES UNDER MULTIPHASE FLOW
}

\author{
V. I. Pegova)* and I. Yu. Moshkin ${ }^{\text {b) }}$ \\ South Urals Federal Research Center of Mineralogy and Geoecology, \\ Ural Branch of the Russian Academy of Sciences, \\ Ilmen State Nature Reserve, Bldg. 1, Miass, 456300, Russian Federation \\ Academician V. P. Makeyev State Rocket Centre JSC, \\ 1, Turgoyakskoye Hwy., Miass, 456300, Russian Federation \\ a) $\otimes$ ofpat@mail.ru;
b) $\otimes$ moshkin_igor@mail.ru \\ *Corresponding author. E-mail: ofpat@mail.ru \\ Address for correspondence: pr. Oktyabrya, 16, Room 103, OFPAT, 456318, Miass, Russian Federation \\ Tel.: +7 (351) 3288550
}

Numerical simulation of the underwater motion of flight vehicles launched from underwater is performed. The updated method of plane sections is used to determine the hydrodynamic parameters of flight vehicles under multiphase flow. Hydrodynamic loading can be evaluated through the determination of nonstationary boundaries of a gas cavity and the linear load on the water-flown aft. By the method of plane sections, the 3D boundary value problem of the cavitational flow of a flight vehicle at an attack angle resolves itself into a plane hydrodynamic problem, separate for each section of the cavity. The predicted results are compared with the experimental data. Validation and verification were performed by comparing the analysis results with the experimental data. The applicability of the method of plane sections to the determination of the hydrodynamic parameters of flight vehicles under multiphase flow is demonstrated.

Keywords: multiphase flow, cavitation, cavity, method of plane sections, mathematical and physical simulation.

\section{Acknowledgment}

The work was performed with the support from the South Urals Federal Research Center of Mineralogy and Geoecology (Ural Branch of the Russian Academy of Sciences) and the Academician V. P. Makeyev State Rocket Centre JSC under the state assignment to SU FRC MG UB RAS, theme No. 0431-2014-0001.

\section{References}

1. Degtiar V.G., Pegov V.I. Results of tryouts of cavitational launch technique for missiles. Raketno-kosmicheskaya Tekhnika, 1999, iss. 1 (43), part 2, pp. 20-35. (In Russian).

2. Poletaev B.I. Mathematical model of motion dynamics of a missile in the underwaters part of the missile cavitational launch technique. In: Problemy mashinovedeniya $i$ mashinostroyeniya [Problems of Machine Science and Mechanical Engineering]. - SPb. : SZGZTU Publ., 2001. Iss. 24. - P. 24-37. (In Russian). 
3. Logvinovich G.V. Gidrodinamika techeny so svobodnymi granitsami [Hydrodynamics of Flows with Free Boundaries]. Kiev, Naukova Dumka Publ., 1969, 216 p. (In Russian).

4. Logvinovich G.V., Buyvol V.N., Dudko A.S., Putilin S.I., Shevchuk Yu.R. Techeniya so svobodnymi poverkhnostyami [Flows with Free Surfaces]. Kiev, Naukova Dumka, 1985. - 178 p. (In Russian).

5. Kapankin E.N., Gulnev S.I. Cavitation flow over a body by a vertical fluid stream. In: Krylov Recitations: Reports. Leningrad, 1973, pp. 14-26. (In Russian).

6. Degtyar V.G., Pegov V.I. Gidrodinamika podvodnogo starta raket [Hydrodynamics of Underwater Missile Launching]. Moscow, Mashinostroenie Publ., 2009, 448 p. (In Russian).

7. Degtiar V.G., Murashev V.I., Perekhodyuk V.N. Solving problems of lateral loads at SLBM launch. In: RKT Collected Papers. Series XIV, 2000, iss. 2 (45), pp. 140-151. (In Russian).

8. Degtiar V.G., Moshkin I.Yu., Pegov V.I., Kalashnikov S.T., Khlybov V.I. $R U$ CNC_RNSG 2015613842 (08 Sept. 2014). (In Russian).

9. Kostin G.F., Degtiar V.G., Kalashnikov S.T., Savelyev V.N., Tyumentsev V.A., Khlybov V.I., Shvaleva R.K. Experimental-analytical methodology used to define parameters of sandygrained roughness on burned surfaces of composite thermal-protective materials. Konsruktsii iz Kompozitsionnykh Materialov, 2018, no. 3, pp. 29-39. (In Russian).

10. Kostin G.F., Zakharyevich D.A., Kalashnikov S.T., Savelyev V.N., Shvaleva R.K. Methodology and results of evaluation of variations in thermal and physical characteristics of a carbon fiber composite on the basis of a phenol-formaldehyde binding agent under heating and decomposition. Konsruktsii iz Kompozitsionnykh Materialov, 2018, no. 4, pp. 63-70. (In Russian).

11. Kalashnikov S.T., Mokin Yu.A., Shvaleva R.K. Design and experimental study of changes in the shape of small lateral surface distortions during ablation of carbon-composite thermal protection of the reentry vehicle model. Konsruktsii iz Kompozitsionnykh Materialov, 2018, no. 4 (152), pp. 42-47. (In Russian).

12. Mokin Yu.A., Kalashnikov S.T., Shvaleva R.K. Estimate of variance of disturbance aerodynamic normal force coefficient of rotary body with small irregular surface distortions of composite thermal protection coating at supersonic flow. Konsruktsii iz Kompozitsionnykh Materialov, 2020, no. 3, pp. 3-8. (In Russian). 
Подана в журнал: 16.06.2020

УДК 621.455: 629.76.085.5

DOI: $10.17804 / 2410-9908.2020 .4 .048-061$

\title{
ПРИМЕНЕНИЕ МЕТОДА ПЛОСКИХ СЕЧЕНИЙ ДЛЯ ОПРЕДЕЛЕНИЯ ХАРАКТЕРИСТИК ЛЕТАТЕЛЬНЫХ АППАРАТОВ ПРИ МНОГОФАЗНОМ ОБТЕКАНИИ
}

\author{
В. И. Пегов ${ }^{\text {a)* }}$, И. Ю. Мошкин ${ }^{\text {) }}$ \\ ${ }^{1}$ Южно-Уральский федеральный научный цеентр минералогии и геоэкологии \\ Уральского отделения Российской академии наук, \\ к. 1, Ильменский заповедник, 456300, г. Миасс, Российская Федераиия \\ ${ }^{2}$ АO «Государственный ракетный иентр имени академика В.П. Макеева», \\ 1, Тургоякское шоссе, 456300, г. Миасс, Российская Федерачия \\ а) ofpat@mail.ru;
б) moshkin_igor@mail.ru \\ *Ответственный автор. Электронная почта: ofpat@mail.ru \\ Адрес для переписки: пр. Октября, 16, к. 103, ОФПАТ, 456318, г. Миасс, Российская Федерация \\ Тел.: +7 (351) 328-85-50
}

Проведено численное имитационное моделирование движения под водой летательных аппаратов, стартующих из-под воды. Применена модернизированная методика плоских сечений для определения гидродинамических характеристик летательных аппаратов при многофазном обтекании. Вычисление гидродинамических нагрузок заключается в определении нестационарных границ газовой каверны и погонной нагрузки на замытую кормовую часть. Трехмерная краевая задача о кавитационном обтекании летательного аппарата под углом атаки с помощью метода плоских сечений сводится к плоской гидродинамической задаче отдельно для каждого сечения каверны. Результаты расчетов сравниваются с экспериментальными данными. Валидация и верификация проведены путем сравнения результатов расчетов с опытными данными. Показана возможность использования модернизированного метода плоских сечений для определения гидродинамических характеристик летательных аппаратов при многофазном обтекании.

Ключевые слова: многофазное обтекание, кавитация, каверна, метод плоских сечений, математическое и физическое моделирование.

\section{1. Введение}

При большой скорости движения под водой летательных аппаратов, стартующих изпод воды, возможно возникновение кавитации корпуса: у стенок обтекаемого тела вследствие местного понижения давления в потоке происходит вскипание воды. Газовые каверны на аппарате формируются также при прохождении аппаратом образующихся при старте газовых полостей или искусственным путем за счет подачи в область разрежения на теле воздуха или иного газа. С возникновением кавитации нарушается сплошность потока и изменяется силовое воздействие жидкости на тело. При совпадении направления силы тяжести и направления нисходящего вертикального потока возникают новые закономерности кавитационных течений, а образующиеся при этом каверны носят название вертикальных каверн. Для снижения гидродинамических нагрузок при выходе из контейнера и обеспечения устойчивого движения аппарата на подводном участке траектории применяют искусственную кавитацию, когда на корпус тела подводят газ из задонного объема через кольцевой зазор контейнера или установленного на аппарате газогенератора, а положение образующейся газовой 
полости (каверны) фиксируют относительно корпуса путем организации схода струй воды с его насадка. Этот способ снижения нагрузок реализован на ряде изделий.

В процессе создания летательных аппаратов, которые движутся на подводном участке траектории в режиме кавитации в вертикальном направлении, проведены широкие экспериментальные и теоретические исследования кавитационного обтекания изделий в условиях значительного изменения вдоль оси каверны гидростатического давления. Для этого выполнены исследования кавитационного обтекания моделей вертикальным нисходящим потоком при различных углах атаки, баллистические испытания моделей аппарата в гидробассейнах и открытых водоемах и запуски полноразмерных макетов летательных аппаратов в натурных условиях. Изучены форма каверны, унос газа из каверны, зависимости гидродинамических характеристик летательного аппарата от реализующихся при старте параметров каверн на участках движения аппарата в контейнере, в воде и при выходе из воды. Газ для образования каверны может поступать из пузыря, образующегося у верхнего среза контейнера, или подаваться из носовых газогенераторов аппарата; чаще используется комбинированный способ захвата газа из пузыря и работы носового газогенератора (рис. 1). Использование того или иного способа образования и развития каверны зависит как от схемы старта, так и от параметров уже выбранной схемы - скорости движения аппарата при выходе из пузыря и на подводном участке, размеров кавернообразующего насадка, количества газа, поступающего в пузырь для образования начальной каверны, интенсивности его подачи и др.

Одной из наиболее сложных проблем, возникающих при реализации кавитационного старта, является задача определения гидродинамических нагрузок. При решении этой задачи возникают значительные математические трудности вследствие сложности протекающих физических процессов. Поэтому значения гидродинамических сил и моментов определяются в основном по результатам баллистических и весовых испытаний моделей, испытаний макетов изделия с плавстенда и подводного носителя. Задача расчетно-теоретического определения гидродинамических характеристик летательного аппарата при кавитационном способе до настоящего времени остается актуальной. Для решения задачи необходимо определить изменяющуюся во времени форму границ каверны и рассчитать погонную нагрузку при смыкании границ каверны на корпус аппарата.

Цели работы - разработка метода определения гидродинамических характеристик летательного аппарата при многофазном обтекании на подводном участке траектории; сведение трехмерной краевой задачи о кавитационном обтекании летательного аппарата под углом атаки с помощью метода плоских сечений к плоской гидродинамической задаче отдельно для каждого сечения каверны; проведение сравнения расчетов с экспериментальными данными.

\section{2. Материал и методика}

Старт летательного аппарата из-под воды широко внедрен у нас и за рубежом с 60-х гг. При этом на подводном участке траектории аппараты испытывают наибольшие нагрузки, что приводит к повышенным требованиям к прочности аппарата и его стабилизации. В результате проведенных исследований было установлено, что наиболее эффективное снижение стартовых нагрузок можно достичь при переходе от режима сплошного обтекания к кавитационному обтеканию аппарата. Кавитационный способ был реализован на летательном аппарате РСМ-52 (ГРЦ Макеева) [1] и РСМ-45 (КБ «Арсенал») [2]. Кавитационное обтекание аппарата достигается формированием каверны при установке на носке аппарата кавитатирующего устройства и источника наддува каверны - газогенератора, так что верхняя часть аппарата размещается в газовой каверне и на нее практически не действуют гидродинамические силы.

В исходном положении (рис. $1 a$ ) аппарат 1 с размещенными на его носовой части квитирующим насадком 2 и газогенератором 3 находится в контейнере 4, на корме аппарата 3 закреплен обтюратор 5, частично перекрывающий проходное сечение кольцевого зазора. 
В контейнере располагается пороховой аккумулятор давления 6, верхний срез контейнера загерметизирован мембраной, старт аппарата осуществляется из незатопленнго водой контейнера. После запуска порохового аккумулятора давление в контейнере быстро возрастает, при достижении избыточного давления в контейнере происходит вскрытие мембраны и над верхним срезом контейнера образуется газовый пузырь 7. При прохождении газового пузыря за квитирующим насадком формируется газовая каверна 8, которая защищает аппарат от набегающего потока воды. Длина каверны при движении в воде постепенно растет как за счет поступления газов газогенератора, так и за счет естественного расширения газа, находящегося в каверне, в соответствии с уменьшением гидростатического давления при вертикальном движении аппарата.

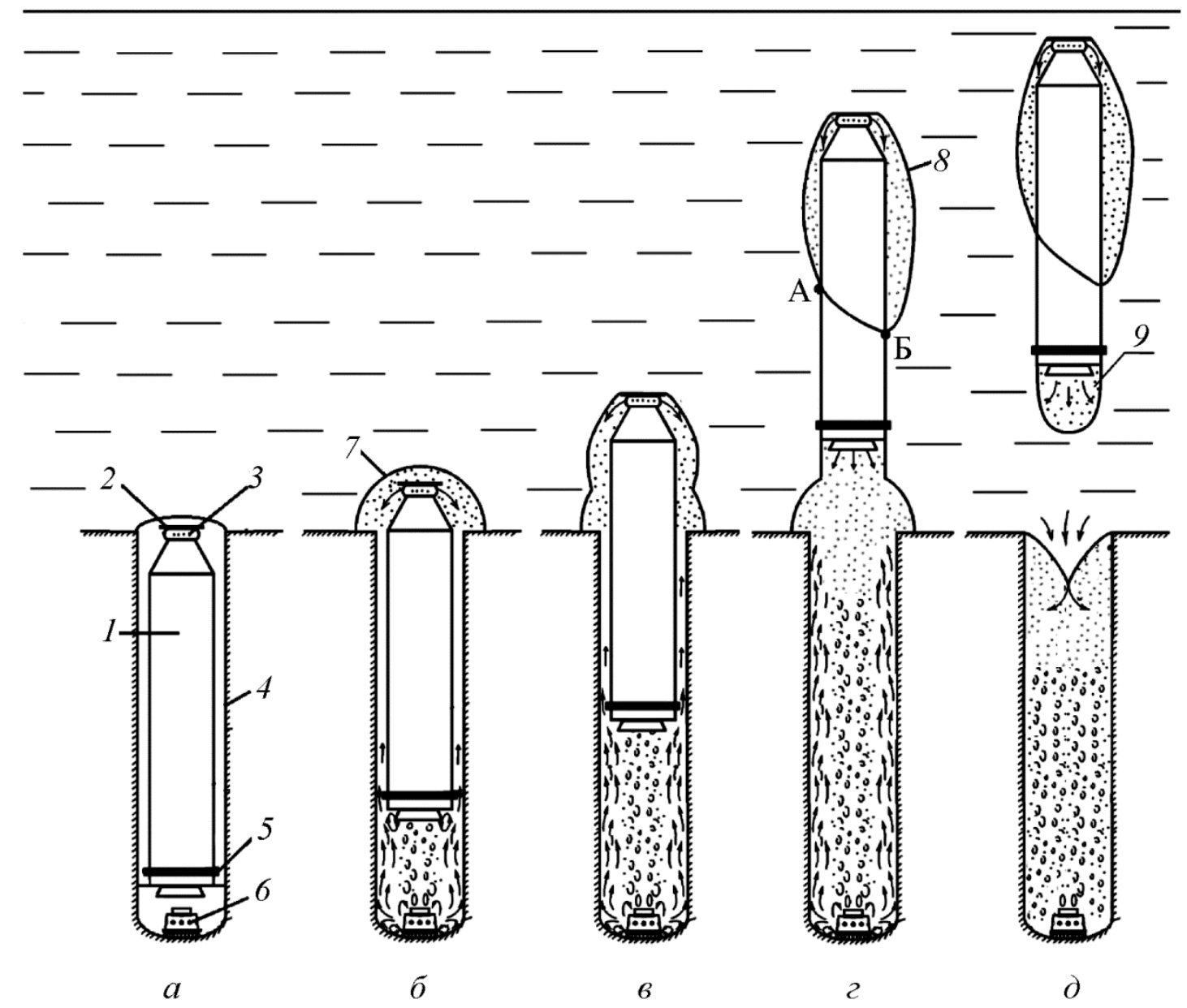

Рис. 1. Схема обтекания аппарата при кавитационном способе движения: 1 - летательный аппарат; 2 - кавитирующий насадок; 3 - газогенератор наддува каверны; 4 - контейнер;

5 - обтюратор; 6 - пороховой аккумулятор давления; 7 - газовый пузырь;

$$
8 \text { - носовая каверна; } 9 \text { - донная каверна }
$$

Согласно гипотезе о «независимом расширении» поперечных сечений каверны трехмерная задача о кавитационном обтекании аппарата под углом атаки сводится к плоской гидродинамической задаче отдельно для каждого сечения $[3,4]$. Однако этот метод нельзя непосредственно использовать в нашем случае, когда в потоке жидкости кроме каверны присутствует за кавитирующим насадком еще и корпус аппарата. Конкретное поперечное сечение каверны может либо пересекаться с телом, либо нет, либо содержать его внутри себя. Это приводит дополнительно к задаче определения погонной нагрузки при пересечении границами каверны цилиндрического корпуса аппарата (рис. 1 2, d). 
При расчете формирования каверны всю область, заполненную жидкостью, разобьем на жидкие слои горизонтальными плоскостями (рис. 1). В жидком слое радиус сечения каверны растет после прохождения его кавитирующим насадком, достигает своего наибольшего значения, а затем уменьшается. Расчет каверны будем проводить в каждом жидком слое согласно принципу «независимости расширения» сечения каверны $S_{\kappa}[3,5]$ :

$$
S_{\kappa}(\xi, t)=S_{H}+\frac{2 \pi}{a} C_{x 0} R_{H} V_{\xi}\left(t-t_{\xi}\right)-\frac{4 \pi C_{x 0}}{a^{2} \rho} \int_{t_{\xi}}^{t} \int_{t_{\xi}}^{u} \Delta P(\xi, \vartheta) d u d \vartheta
$$

где $S_{\kappa}=p r_{\kappa}^{2}, r_{\kappa}$ - радиус каверны, $\Delta P(\xi, \vartheta)=P_{\infty}-P_{\kappa}(\vartheta)-\rho g \xi ; P_{\infty}$ и $P_{\kappa}-$ давления в воде на уровне среза контейнера и в каверне; $C_{x 0}, S_{H}, R_{H}-$ коэффициент сопротивления, площадь и радиус насадка соответственно; $\xi, t \xi, V_{\xi}$ - соответственно координата сечения, время и скорость его прохождения насадком; $\rho$ - плотность воды; $a$ - эмпирический коэффициент $(a=1,5 \ldots 1,9)$.

Для упрощения расчетов введем интегралы

$$
J_{0}(u)=\frac{4 \pi C_{x 0}}{a^{2} \rho} \int_{0}^{u} P_{\kappa}(\vartheta) d \vartheta ; \quad J_{1}(t)=\int_{0}^{t} J_{0}(u) d u
$$

которые имеют постоянный и одинаковый нижний предел. Тогда уравнение (1) запишется в виде

$$
\begin{aligned}
S_{\kappa}(\xi, t)=S_{H}+ & \frac{2 \pi}{a} C_{x 0} R_{H} V_{\xi}\left(t-t_{\xi}\right)-\frac{2 \pi C_{x 0}}{a^{2} \rho}\left(P_{\infty}-\rho g \xi\right)\left(t-t_{\xi}\right)^{2}+ \\
& +J_{1}(t)-J_{1}\left(t_{\xi}\right)-\left(t-t_{\xi}\right) J_{0}\left(t_{\xi}\right)
\end{aligned}
$$

где $J_{0}\left(t_{\xi}\right), J_{1}\left(t_{\xi}\right), J_{1}(t), V_{\xi}$ и $t \xi$ выбираются из таблиц, организованных в процессе расчета.

При расчете продольного движения аппарата в контейнере будем рассматривать равноускоренное движение $\left({ }_{p}=\right.$ const $)$, при котором осевая перегрузка аппарата будет минимальной. Требуемый массовый расход порохового аккумулятора давления при равноускоренном движении аппарата в контейнере и гидростатическом давлении $P_{\infty}$ находится по формуле

$$
n k_{\text {ПАД }}=n \&_{\text {ок. }}+\otimes_{p} m_{0}\left(\frac{1}{x_{p}+\tau_{0 \text { нач. }} / v S_{u}}+\frac{\rho\left(C_{x}-g\right)}{(v-\chi) k P_{\infty}}\right),
$$

где $m_{0}$ - текущая масса газа в подаппаратном объеме; $\chi=S_{\text {обт }} / S_{u} ; S_{\text {обт }}$ - проходное сечение в обтюраторе; $S_{u}$ - площадь сечения цилиндра.

Используя рассчитанные формы каверны и области смыкания границ каверны на корпус аппарата, перейдем к задаче определения погонной нагрузки. Кавитирующий насадок, проходя сквозь плоскость жидкости, порождает элемент каверны, который расширяется по инерции, преодолевая внешнее избыточное давление. Через некоторый промежуток времени запас кинетической энергии исчерпывается, поперечное сечение каверны достигает максимальной площади, после чего начинает постепенно сужаться. Сечение каверны, кроме того, 
испытывает сопротивление жидкости и сносится поперечным потоком. Наконец наступает такой момент, когда сечение каверны коснется с наветренной стороны поверхности тела (рис. 1 2, сеч. А) и начнется несимметричное смыкание каверны на цилиндрическую поверхность тела. Обычно форма кавитирующего насадка близка к диску и при вертикальном движении тела поперечное сечение каверны на протяжении всего своего развития, вплоть до момента начала смыкания на тело, остается близким к круговому, а скорость сноса его близка к скорости подводного носителя $U$.

Решение будем искать в конечной области, а именно - в кольцевом слое, ограниченном в начальный момент снаружи круговой цилиндрической поверхностью $S_{2}$ радиуса $R_{20}$, а изнутри - поверхностью $S_{1}$ радиуса $R_{10}\left(R_{10}<R_{20}\right)$, равного в начальный момент радиусу каверны. На рис. 2 представлена схема течения в кольцевом слое и принятые обозначения при решении задачи. Кроме того, будем считать жидкость идеальной невесомой и несжимаемой, а вызванное течение жидкости - потенциальным. На поверхности каверны $S_{l}$, как обычно, должно выполняться условие постоянства давления

$$
\left.P\right|_{S_{1}}=P_{\kappa}(t)
$$

где $P_{\kappa}(t)$ - давление газов в каверне. При $t>0$ на поверхности $S_{1}$ возникает поверхность контакта тела с жидкостью, размеры которой будут постепенно расти и определяться величиной центрального угла $2 \theta_{c}$. На смоченной части поверхности тела $S_{T}$ должно выполняться условие непротекания, т.е. производная от искомого потенциала скоростей $\varphi$ по нормали должна удовлетворять равенству

$$
\left.\frac{\partial \varphi}{\partial n}\right|_{S_{T}}=U \cos \theta, \text { при }-\theta_{C} \leq \theta \leq \theta_{C} .
$$

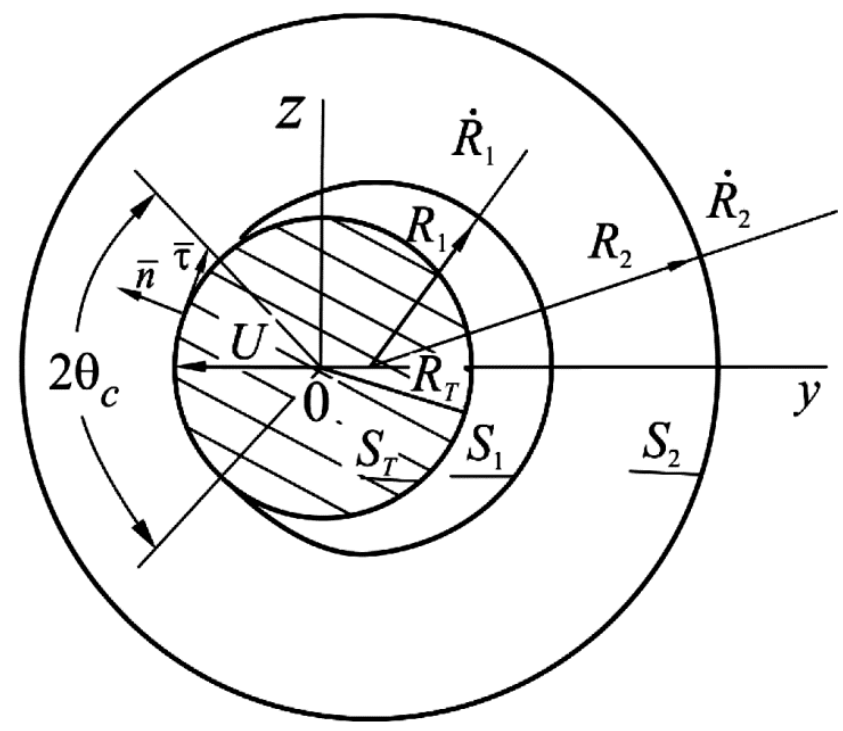

Рис. 2. Схема течения и принятые обозначения в кольцевом слое

В качестве внешней ограничивающей поверхности $S_{2}$ примем некоторую свободную поверхность, аналогичную, например, наблюдаемой в гидродинамических трубах с открытой рабочей частью. Граничное условие на этой свободной поверхности невесомой и несжимаемой жидкости можно записать в виде 


$$
\left.\frac{\partial \varphi}{\partial t}\right|_{S_{2}}=\frac{1}{2} v_{2}^{2}(t, \theta)
$$

где $v_{2}(t, \theta)$ - абсолютная скорость жидкости на свободной поверхности. Проводя интегрирование последнего уравнения по времени, найдем для каждого момента времени распределение потенциала на поверхности $S_{2}$

$$
\left.\varphi\right|_{S_{2}}=\varphi_{2}(t, \theta)
$$

Таким образом, теоретическое решение поставленной задачи сведено к нахождению в кольцевом слое $R_{10} \leq r \leq R_{20}$ потенциала скоростей $\varphi$, удовлетворяющего уравнению Лапласа и граничным условиям (5) - (7). Эта краевая задача решена в работах $[6,7]$ аналитическим методом с помощью разложения в ряд искомой гармоничной функции $\varphi$. Аналитическое решение получено в виде зависимостей погонной нагрузки $\frac{d C_{y}^{\sigma}}{d \bar{x}}$ от кинетических параметров в кольцевом слое

$$
\frac{d C_{y}^{\alpha}}{d \bar{x}}=\frac{4 \alpha}{\pi}\left[\left(\cos \theta_{c}-\hat{R}_{1}^{\alpha}\right) \cos \theta_{c} \hat{\theta}_{c}^{\alpha}-R_{1}^{\alpha} \sin \theta_{c}\right] .
$$

А также значение коэффициента $C_{y}^{\sigma}$ как результат интегрирования по замытым на корпус слоям

$$
C_{y}^{\alpha}=\frac{1}{\pi}\left(2 \tilde{\theta}_{c}+\sin 2 \tilde{\theta}_{c}-4 \tilde{R}_{1 H}^{\alpha} \sin \tilde{\theta}_{c}\right)
$$

Здесь значения $\tilde{\theta}_{c}$ и $\hat{R}_{1 \mu}^{e}$ берутся в нижнем кольцевом слое пересечения границ каверны, например точка Б (рис. 1 г). Из последнего равенства следует, что при полном смыкании нижнего слоя на цилиндр $\tilde{\theta}_{c}=\pi$, коэффициент $C_{n}^{\alpha}=2,0$. Этот результат совпадает с известным результатом Г.А. Логвиновича, когда тело обтекается в режиме частичной кавитации [3].

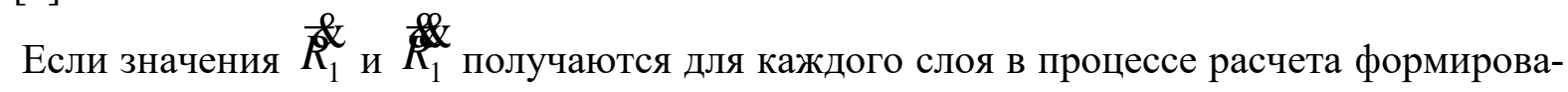
ния каверны, то $\mathcal{E}_{c}^{\ell}$ рассчитываем по формуле [6]:

$$
\bar{\theta}_{c}^{\mathrm{e}}=\frac{d \theta_{c}}{d \bar{t}}=\sum_{n=1}^{\infty} \bar{k}_{n}^{(1)} \sin n \theta_{c}
$$

где $\bar{k}_{n}^{(1)}-$ коэффициент из аналитического решения; $\bar{t}=\frac{t U}{R_{T}}-$ безразмерное время. 
В формулах (8), (9) и (10) приняты следующие безразмерные величины: $C_{y}^{\alpha}=\frac{2 y}{\pi R_{T}^{2} \rho U V} ; y-$ боковая нагрузка на аппарат; $V-$ продольная скорость $; \bar{y}=\frac{y}{R_{T}}, \bar{x}=\frac{\bar{y}}{\alpha}$; $\alpha=\frac{U}{V} ; \hat{R}_{1}^{\&}=\frac{R_{1}^{\&}}{U} ; K_{1}^{\mathbb{R}}=\frac{R_{T}}{U^{2}} ; \bar{k}_{n}^{(1)}=\frac{k_{n}^{(1)}}{U} ; \hat{\theta}_{c}^{\&}=\frac{R_{T}}{U} \frac{d \theta_{c}}{d t}$.

При кавитационном способе старта, как и при других способах, наибольшие изгибающие гидродинамические моменты возникают во время выхода аппарата из контейнера под действием поперечного потока жидкости, скорость которого определяется скоростью подводного носителя. Сравнительный анализ уровня нагрузок для различных способов старта можно определить безразмерным импульсом гидродинамического момента, действующего на аппарат за время выхода из контейнера $\bar{J}_{m z}$. На рис. 3 представлен безразмерный импульс момента $\bar{J}_{m z}$ поперечной нагрузки относительно оси, лежащей в плоскости обтюратора, за время выхода модели из контейнера, при $\bar{u}=0,16$ зависимости от безразмерной длины каверны $\bar{l}_{K}$ :

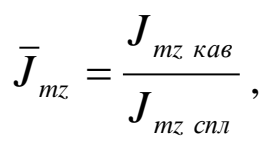

где $J_{m z \text { кав }} ; J_{m z \text { сnл }}-$ импульсы момента $m_{z}\left(m_{z}=C_{y}^{\alpha} \cdot \alpha\left(1-\bar{l}_{K}\right)\right)$ при кавитационном и сплошном обтекании; $\bar{u}=U / V_{p u} ; U$ - скорость подводного носителя; $V_{p u}$ - скорость выхода аппарата из контейнера; $\bar{l}_{K}=l_{K} / L ; l_{K}$ - длина каверны; $L$ - длина аппарата. Из графика рис. 3 следует, что при $\bar{l}_{K}=0,8$ безразмерный импульс момента при кавитационном старте составляет примерно десятую часть импульса при обычном старте $\left(\bar{l}_{K}=0\right)$. Это свидетельствует о возможности достижения высокой эффективности кавитационного способа старта за счет длины каверны.

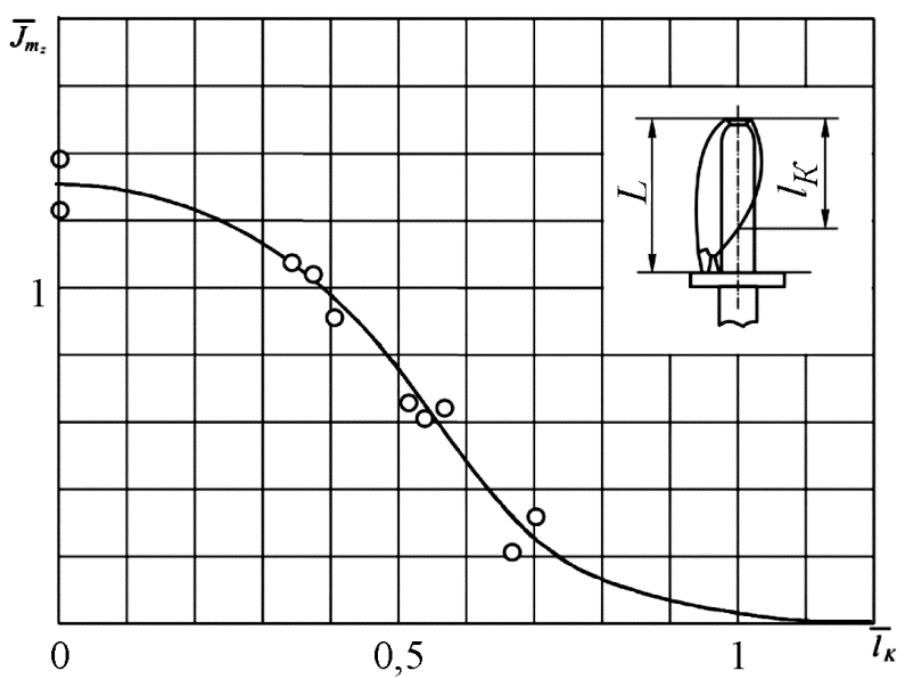

Рис. 3. Зависимость безразмерного импульса момента $\bar{J}_{m z}$ от безразмерной длины каверны $\bar{l}_{K}$ : сплошная линия - расчет; значки - эксперимент 
В заключение отметим, что при известных зависимостях от времени коэффициента $C_{y}^{\alpha}$ и длины каверны $l_{K}$, пользуясь гипотезой плоских сечений, можно найти и все оставшиеся коэффициенты, например коэффициент вращательных производных $C_{y}^{w_{z}}=C_{y}^{\alpha}\left(1-\bar{x}_{\text {и.м. }}\right)$, поперечную присоединенную массу $\lambda_{22}=\rho \pi R_{T}^{2}\left(1-\bar{l}_{K}\right)$ и др. [6].

\section{3. Результаты и обсуждение}

В данном разделе приводятся результаты экспериментальных исследований моделей в гидротрубах, полученные в АО «Государственный ракетный центр имени академика В.П. Макеева» во время проведения научно-исследовательских и опытно-конструкторских работ. На рис. 4 представлены фотографии картины кавитационного обтекания модели. Для каждой фотографии указаны значения числа кавитации, угла атаки, относительного диаметра диска. Фотографии на рис. 4 (в двух проекциях) позволяют получить информацию о размерах и форме замытой водой части модели. На рис. 5 и 6 представлены зависимости коэффициента сопротивления $C_{x}$ и коэффициента нормальной силы $C_{y}$ от числа кавитации $\sigma$ и угла атаки $\alpha$. Анализ картины течения позволяет выделить три основных режима кавитационного обтекания, характеризующихся различным взаимным положением модели и каверны и соответственно своими особенностями гидродинамических характеристик. На первом режиме - режим бесконтактного обтекания - модель полностью находится внутри каверны, с жидкостью взаимодействует только кавернообразующий насадок. В этом случае сопротивление и нормальная сила тела равны сопротивлению и нормальной силе кавернообразующего насадка. Для насадка в виде диска, установленного перпендикулярно оси аппарата, коэффициент сопротивления $C_{x}$ равен

$$
C_{x}=0,82(1+\sigma) \cos \alpha \bar{d}_{H}^{2}
$$

а коэффициент нормальной силы равен нулю $\left(C_{y}=0\right)$. После касания хвостовой частью модели границ каверны происходит переход ко второму режиму обтекания, при котором корпус модели пересекает границу каверны. Увеличение числа кавитации $\sigma>\sigma_{\max }$ приводит к дальнейшему уменьшению размеров каверны, длина каверны становится меньше длины тела, хвостовая часть каверны смыкается на корпусе тела, что характерно для третьего режима обтекания - обтекания с частичной кавитацией. Переход от второго к третьему режиму обтекания сопровождается быстрым падением несущих свойств летательного аппарата.

Полученная система уравнений (раздел 2) была реализована в программном комплекce CNC_RNSG_[8], разработанном Южно-Уральским федеральным научным центром минералогии и геоэкологии УрО РАН совместно с АО «Государственный ракетный центр имени академика В.П. Макеева» в рамках государственного задания ЮУ ФНЦ МиГ УрО РАН по теме № 0431-2014-0001. Этот программный комплекс был оттестирован на экспериментальных данных, полученных на большой скоростной гидродинамической трубе 23К25. Валидация и верификация проведены путем сравнения результатов расчетов с опытными данными. Программный комплекс зарегистрирован в Роспатенте. Для проверки имитационного моделирования проведено сравнение расчетов с экспериментальными данными, полученными в гидробассейне, где были проведены пуски крупномасштабной модели летательного аппарата из контейнера. 


$$
d_{H} / d=0,55 ; \alpha=2,5^{\circ}
$$
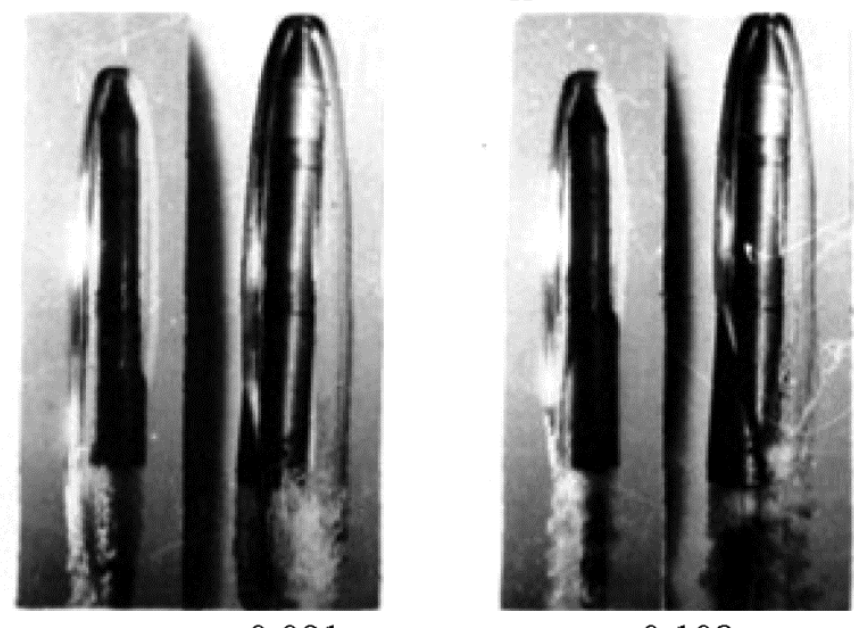

$\sigma=0,108$

$$
d_{H} / d=0,55 ; \alpha=5^{\circ}
$$

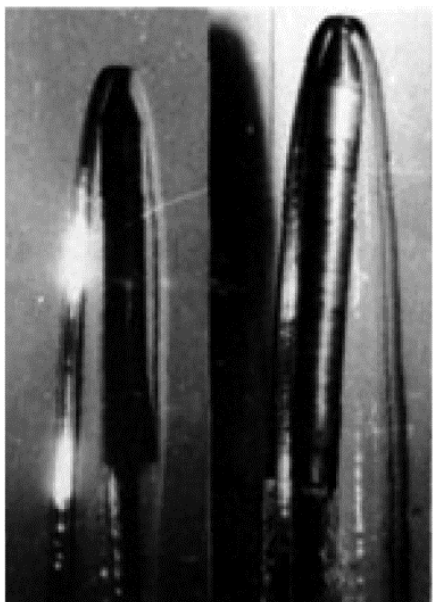

$\sigma=0,055$

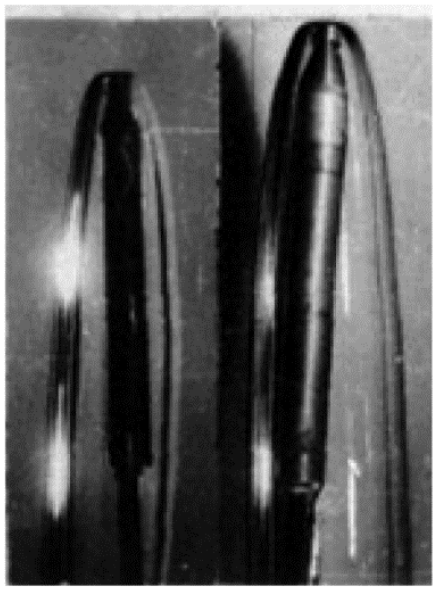

$\sigma=0,0755$

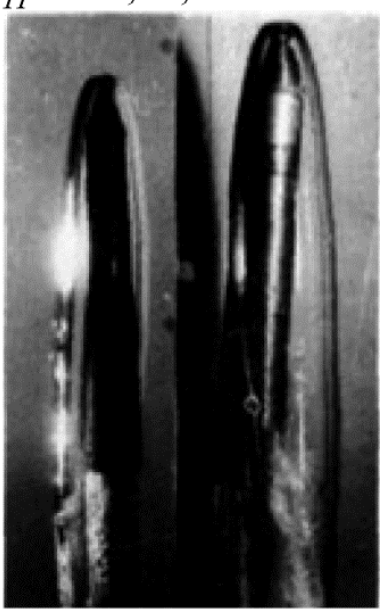

$\sigma=0,087$

$d_{H} / d=0,735 ; \alpha=5^{\circ}$

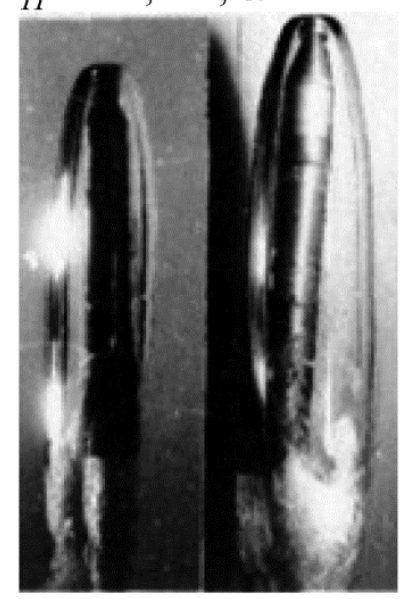

$\sigma=0,115$

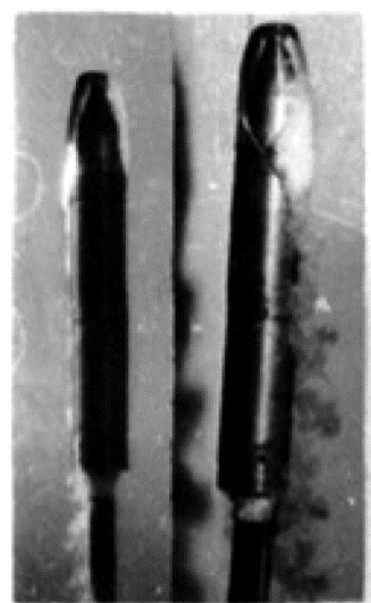

$\sigma=0,203$

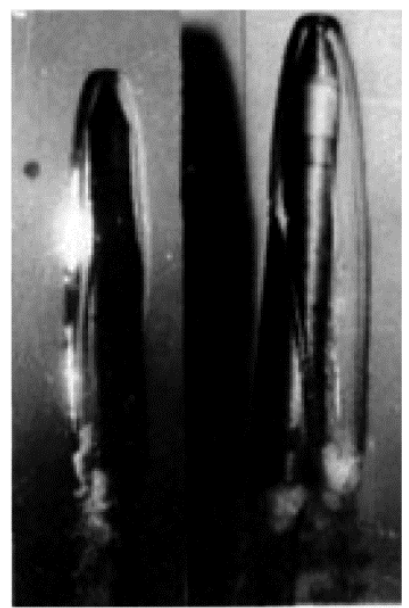

$\sigma=0,107$

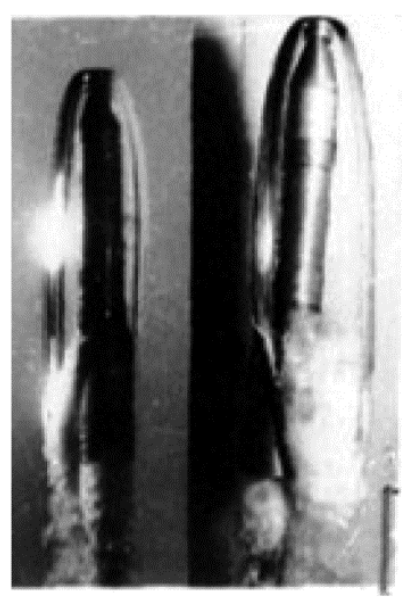

$\sigma=0,131$

Рис. 4. Фотографии кавитационного обтекания модели 


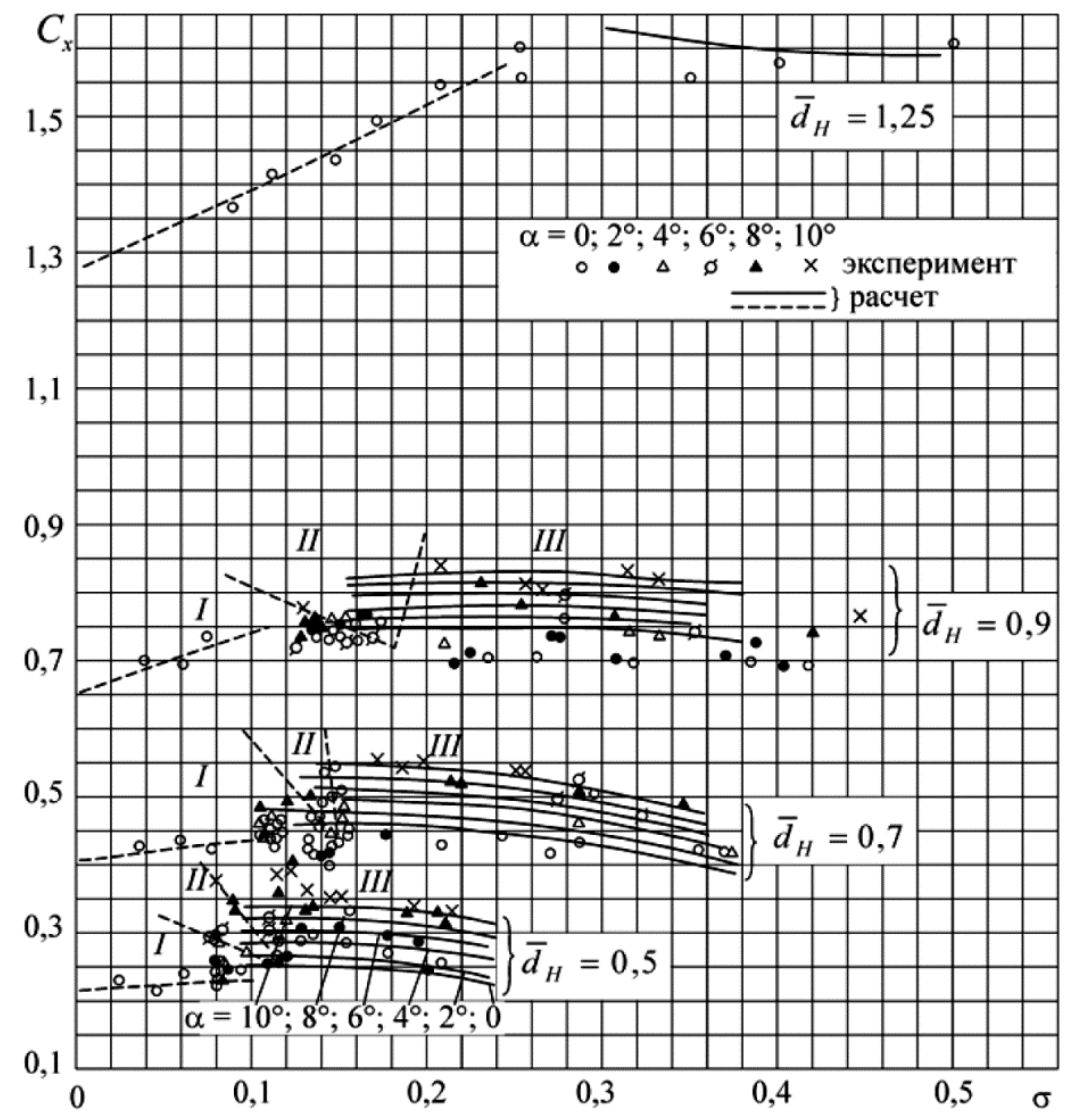

Рис. 5. Зависимости коэффициента сопротивления $C_{x}$ от числа кавитации $\sigma$

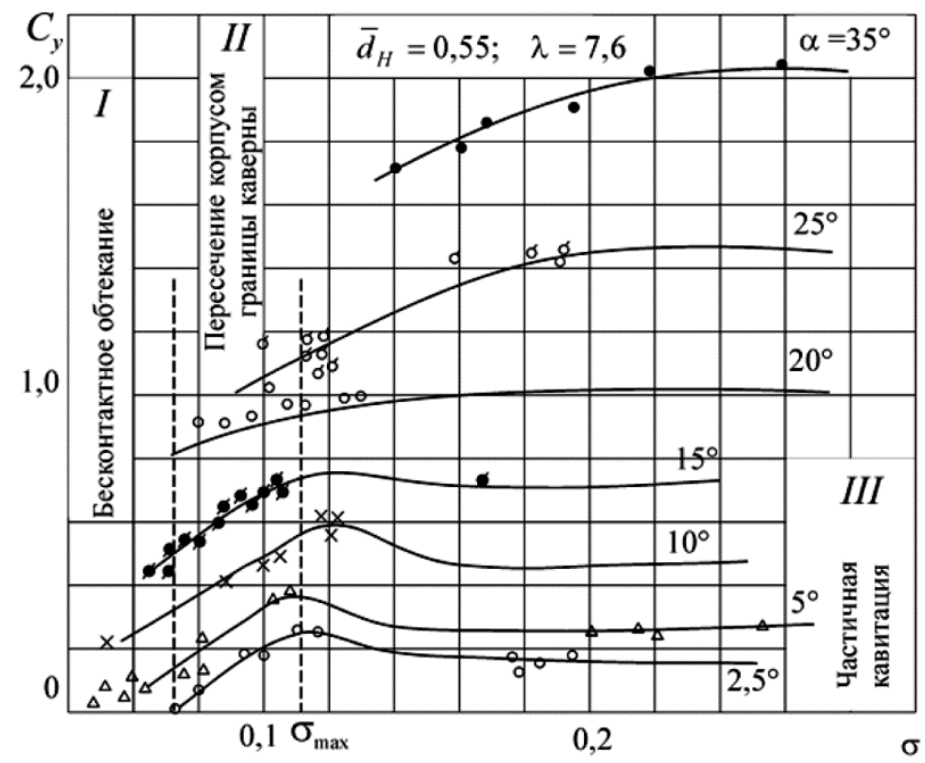

Рис. 6. Зависимость коэффициента нормальной силы $C_{y}$ от числа кавитации $\sigma$

На рис. 7 приведено сравнение расчетных профилей каверн с экспериментальными данными, полученными на экспериментах в большой скоростной гидродинамической трубе, красной линией обозначен профиль каверны, полученный расчетным путем. Результаты предоставлены для трех режимов обтекания: обтекание в суперкаверне, когда летательный ап- 
парат полностью находится в каверне; обтекание при развитой кавитации, когда большая поверхность летательного аппарата находится в каверне; обтекание при частичной кавитации. Моделирование процесса разделения каверны летательного аппарата от газовой полости, образующейся у верхнего среза контейнера, проводилось в гидробассейне. На рис. 8 представлено сравнение расчетных и экспериментальных профилей каверны для крупномасштабной модели летательного аппарата, полученных с помощью видеосъемки в гидробассейне. Расчетные профили каверны удовлетворительно согласуются с экспериментальными (рис. 7 и 8). В работах [9-12] приведены результаты определения характеристик летательного аппарата при многофазном обтекании при больших и гиперзвуковых скоростях при входе в атмосферу.

$$
\mathrm{Fr}_{H}=11,5 ; \quad \alpha=4^{\circ} ; \bar{d}_{H}=0,6
$$

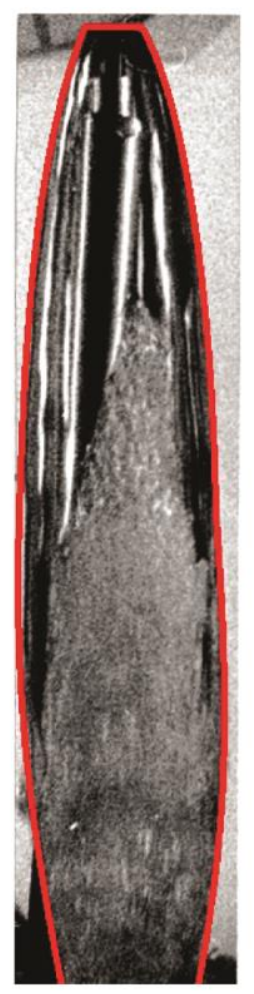

$\sigma_{0}=-0,02$

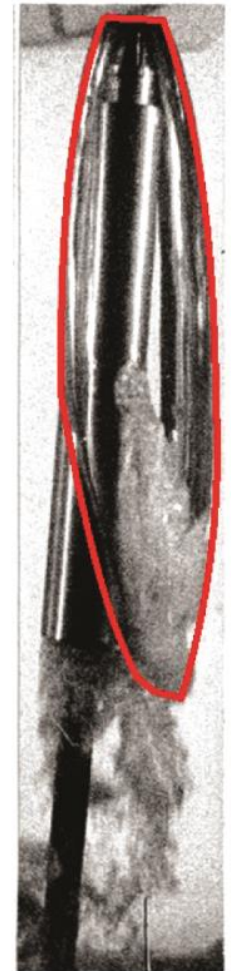

$\sigma_{0}=0,008$

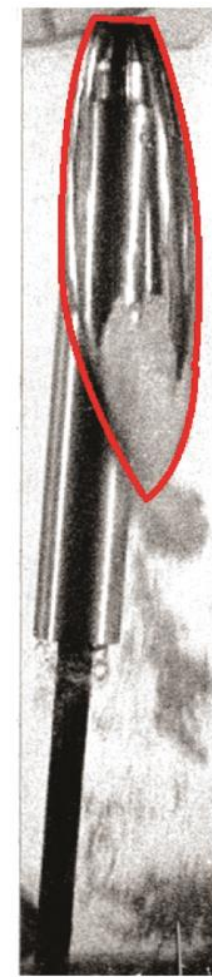

$\sigma_{0}=0,195$
Рис. 7. Сравнение расчетных профилей каверн с экспериментальными данными в большой скоростной гидродинамической трубе, красной линией обозначен профиль, полученный расчетным путем

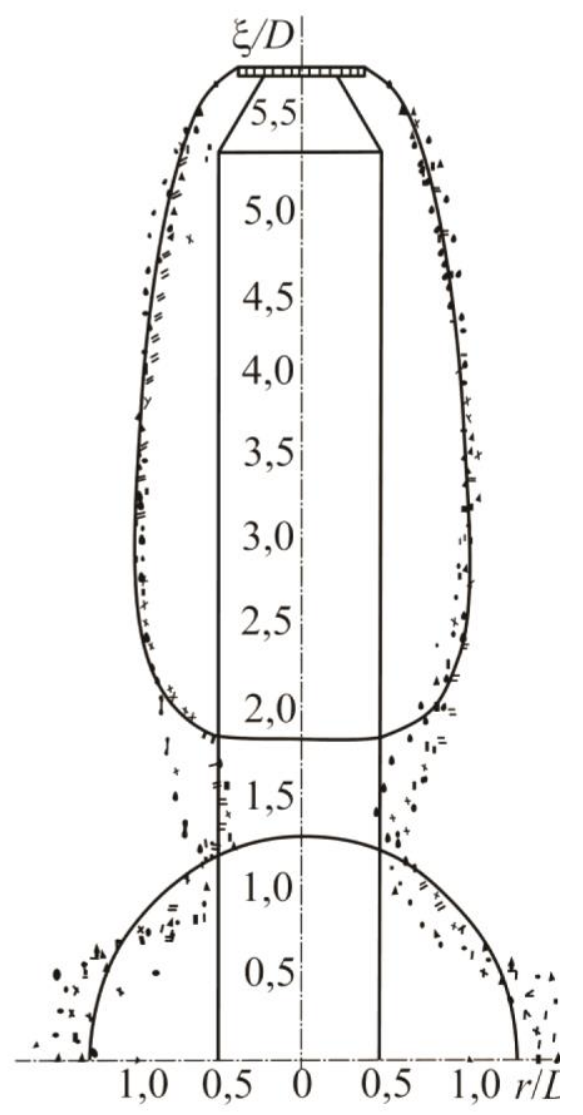

Рис. 8. Сравнение расчетных профилей каверн с экспериментальными, полученныеми в гидробассейне: сплошная линия - расчет; значки - эксперимент

\section{4. Заключение}

На основе метода плоских сечений разработан математический метод определения характеристик летательных аппаратов при многофазном обтекании. Трехмерная краевая задача о кавитационном обтекании летательного аппарата под углом атаки в воде с помощью метода плоских сечений сводится к плоской гидродинамической задаче отдельно для каждого сечения каверны. Результаты расчетов сравниваются с экспериментальными данными. Валидация и верификация проведены путем сравнения результатов расчетов с опытными данными. Полученные расчетные профили каверны удовлетворительно согласуются с экспериментальными. Показана возможность использования модернизированного метода плоских сечений для определения 
гидродинамических характеристик летательных аппаратов при многофазном обтекании. Создан программный комплекс, позволяющий проводить имитационное моделирование движения под водой летательных аппаратов, стартующих из-под воды.

\section{Благодарность}

Работа выполнена при поддержке Южно-Уральского федерального научного центра минералогии и геоэкологии УрО РАН и АО «Государственный ракетный центр имени академика В.П. Макеева» в рамках государственного задания ЮУ ФНЦ МиГ УрО РАН по теме № 0431-2014-0001.

\section{Литература}

1. Дегтярь В. Г., Пегов В. И. Результаты экспериментальной отработки кавитационного способа старта ракет // РКТ. - 1999. - Вып. 1 (43), ч. 2. - С. 20-35.

2. Полетаев Б. И. Математическая модель динамики движения ракеты на подводном участке кавитационного способа старта ракет // Проблемы машиноведения и машиностроения : межвуз. сб. - СПб : СЗГЗТУ, 2001. - Вып. 24. - С. 24-37.

$3 . \quad$ Логвинович Г. В. Гидродинамика течений со свободными границами. - Киев : Наукова думка, 1969. - 216 с.

4. Течения со свободными поверхностями / Г. В. Логвинович, В. Н. Буйвол, А. С. Дудко, С. И. Путилин, Ю. Р. Шевчук. - Киев : Наукова думка, 1985. - 295 с.

5. Капанкин Е. Н., Гульнев С. И. Кавитационное обтекание тел вертикальным потоком жидкости // XXII Всесоюзная науч.-техн. конференция по теории корабля (Крыловские чтения 1973 г.), Ленинград, 1973 г. : краткие тезисы докладов. - Ленинград : Судостроение, 1973. - C. 14-26.

6. Дегтярь В. Г., Пегов В. И. Гидродинамика подводного старта ракет. - М. : Машиностроение, 2009. - 448 с.

7. Дегтярь В. Г., Мурашев В. И., Переходюк В. Н. Решение задач поперечных нагрузок при старте БРПЛ // Ракетно-космическая техника : сборник статей. Серия XIV. - Миасс : ГРЦ Макеева, 2000. - Вып. 2 (45). - С. 140-151.

8. Программа для ЭВM CNC_RNSG : свидетельство о регистрации RU 2015613842 / Дегтярь В. Г., Мошкин И. Ю., Пегов В. И., Калашников С. Т., Хлыбов В. И. - № 2014619029 ; заявл. 08.09.2014 ; опубл. 26.03.2015.

9. Методология экспериментальных и расчетных работ по определению параметров песочно-зернистой шероховатости обгарных поверхностей / Г. Ф. Костин, В. Г. Дегтярь, С. Т. Калашников, В. Н. Савельев, В. А. Тюменцев, В. И. Хлыбов, Р. К. Швалева // Конструкции из композиционных материалов. - 2018. - № 3. - С. 29-39.

10. Методика и результаты оценки изменения теплофизических характеристик углепластика на основе фенолформальдегидного связующего при нагреве и разложении / Г. Ф. Костин, Д. А. Захарьевич, С. Т. Калашников, В. Н. Савельев, Р. К. Швалева // Конструкции из композиционных материалов. - 2018. - № 4. - С. 63-70.

11. Калашников С. Т., Мокин Ю.А., Швалева Р. К. Расчетно-экспериментальное исследование изменения формы малых искажений боковой поверхности в процессе абляции углепластикового теплозащитного покрытия модели спускаемого летательного аппарата // Конструкции из композиционных материалов. - 2018. - Вып.4 (152). - С. 42-47.

12. Мокин Ю. А., Калашников С. Т., Швалева Р. К. Оценка дисперсии коэффициента возмущающей аэродинамической нормальной силы тела вращения с малыми случайными искажениями поверхности композитного теплозащитного покрытия при сверхзвуковом обтекании // Конструкции из композиционных материалов. - 2020. - № 3. - С. 3-8. 